\title{
Hadronic contributions to the muon anomalous magnetic moment
}

\author{
Massimiliano Procura* \\ Fakultät für Physik, Universität Wien, Boltzmanngasse 5, 1090 Wien, Austria \\ E-mail: mprocuraeunivie.ac.at
}

\section{Gilberto Colangelo}

Albert Einstein Center for Fundamental Physics, Institute for Theoretical Physics, University of Bern, Sidlerstrasse 5, 3012 Bern, Switzerland

E-mail: gilbertoditp.unibe.ch

\section{Martin Hoferichter}

Institute for Nuclear Theory, University of Washington, Seattle, WA 98195-1550, USA

E-mail: mhofereuw. edu

\section{Peter Stoffer}

Department of Physics, University of California at San Diego, La Jolla, CA 92093, USA

E-mail: pstoffer@ucsd.edu

The largest uncertainties in the Standard Model calculation of the anomalous magnetic moment of the muon $(g-2)_{\mu}$ come from hadronic effects, namely hadronic vacuum polarization (HVP) and hadronic light-by-light (HLbL) contributions. Especially the latter is emerging as a potential roadblock for a more accurate determination of $(g-2)_{\mu}$. The main focus here is on a novel dispersive description of the HLbL tensor, which is based on unitarity, analyticity, crossing symmetry, and gauge invariance. This opens up the possibility of a data-driven determination of the HLbL contribution to $(g-2) \mu$ with the aim of reducing model dependence and achieving a reliable error estimate.

Our dispersive approach defines unambiguously the pion-pole and the pion-box contribution to the HLbL tensor. Using Mandelstam double-spectral representation, we have proven that the pion-box contribution coincides exactly with the one-loop scalar-QED amplitude, multiplied by the appropriate pion vector form factors. Using dispersive fits to high-statistics data for the pion vector form factor, we obtain $a_{\mu}^{\pi \text {-box }}=-15.9(2) \times 10^{-11}$. A first model-independent calculation of effects of $\pi \pi$ intermediate states that go beyond the scalar-QED pion loop is also presented. We combine our dispersive description of the HLbL tensor with a partial-wave expansion and demonstrate that the known scalar-QED result is recovered after partial-wave resummation. After constructing suitable input for the $\gamma^{*} \gamma^{*} \rightarrow \pi \pi$ helicity partial waves based on a pion-pole lefthand cut (LHC), we find that for the dominant charged-pion contribution this representation is consistent with the two-loop chiral prediction and the COMPASS measurement for the pion polarizability. This allows us to reliably estimate $S$-wave rescattering effects to the full pion box and leads to $a_{\mu}^{\pi \text {-box }}+a_{\mu, J=0}^{\pi \pi, \pi \text {-pole LHC }}=-24(1) \times 10^{-11}$.

XIII Quark Confinement and the Hadron Spectrum - Confinement2018

31 July - 6 August 2018

Maynooth University, Ireland

\footnotetext{
* Speaker.
} 


\section{Introduction}

The anomalous magnetic moment of the muon $(g-2)_{\mu}$ has been measured [1] and computed to very high precision of about $0.5 \mathrm{ppm}$ (see e.g. [2]). For more than a decade, a discrepancy has persisted between the experiment and the Standard Model prediction, now of about $3 \sigma$. Forthcoming measurements at FNAL [3] and J-PARC [4] will update the experimental value with the aim of increasing the precision by a factor of 4 and checking for systematic errors.

The main uncertainty of the theory prediction is due to strong interaction effects [5, 6, 7]. Here the largest contribution is given by the leading-order HVP, whose currently most precise determination relies on a dispersion relation linking it to cross-section measurements for $e^{+} e^{-}$annihilations into hadrons, with the dominant effect arising from $\pi \pi$ intermediate states. Since early determinations, the experimental situation in $e^{+} e^{-} \rightarrow$ hadrons has improved considerably, and keeps doing so thanks to a dedicated program [2], but at the same time the required precision of the HVP has increased, especially in view of the anticipated improved experimental measurement of $(g-2)_{\mu}$. Thus a proper treatment of experimental errors and correlations is becoming crucial and is now the focus of extensive study. This includes radiative corrections, which need to be taken into account properly in order to ensure a consistent counting of higher-order HVP iterations. Most current HVP compilations are based on direct integration of the experimental data $[8,9,10]$, and a novel dispersive approach exploiting global constraints from analyticity and unitarity in the pion vector form factor and $\pi \pi$ scattering [11] provides complementary information to consolidate uncertainty estimates for the $\pi \pi$ channel. Furthermore, very interesting progress has been made in recent years by several lattice collaborations to compute $(g-2)_{\mu}^{\mathrm{HVP}}$ from first principles according to different strategies. For a compilation of results and ongoing efforts in estimating various systematic effects, we refer to [12].

If forthcoming data from $e^{+} e^{-}$experiments and/or progress in lattice calculations help reduce uncertainties in the HVP, the subleading HLbL contribution would dominate the theory error. In present calculations of the HLbL contribution, systematic errors are difficult, if not impossible, to quantify, due to model dependence. A new strategy is required to provide a solid estimate of the theory uncertainties and reduce them. Lattice QCD is making remarkable progress in this direction, and may play a leading role in this field in the near future $[13,14,15,16,17,18,19$, 20, 21]. In [22, 23], we have presented the first dispersive description of the HLbL tensor. ${ }^{1}$ By making use of the fundamental principles of unitarity, analyticity, crossing symmetry, and gauge invariance, we provide an approach that reduces model dependence and allows for a more datadriven determination of the HLbL contribution to $(g-2)_{\mu}$.

Here, we report on a several improvements of our dispersive framework [25, 26, 27, 39]. We have constructed a generating set of Lorentz structures for the HLbL tensor that is free of kinematic singularities and zeros. This simplifies significantly the calculation of the HLbL contribution to $(g-2)_{\mu}$. Within our dispersive formalism, the definitions of both the pion-pole and pion-box topologies are unambiguous. By constructing a Mandelstam representation for the scalar functions, we prove that the box topologies coincide with the scalar-QED (sQED) contribution multiplied by pion vector form factors. Here we present a numerical evaluation of the pion box using a form factor

\footnotetext{
${ }^{1} \mathrm{~A}$ different approach, which aims at a dispersive description of the muon vertex function instead of the HLbL tensor, has been presented in [24].
} 
fit to high-statistics data, in turn using a dispersive representation to analytically continue the timelike data into the space-like region required for the $(g-2)_{\mu}$ integral and show that this contribution can be calculated with negligible uncertainties. We then present a first numerical evaluation of $S$-wave $\pi \pi$-rescattering effects, which unitarize the pion-pole contribution to $\gamma^{*} \gamma^{*} \rightarrow \pi \pi$. This constitutes the first step towards a full treatment of the $\gamma^{*} \gamma^{*} \rightarrow \pi \pi$ partial waves $[28,29,30]$. In particular, our calculation settles the role of the pion polarizability, which enters at next-to-leading order in the chiral expansion of the HLbL amplitude $[31,32,33]$ and has been suspected to produce sizable corrections in [32].

\section{Lorentz structure of the HLbL tensor}

In order to study the HLbL contribution to $(g-2)_{\mu}$, we need a description of the HLbL tensor, namely the hadronic Green's function of four light-quark electromagnetic currents, evaluated in pure QCD:

$$
\Pi^{\mu \nu \lambda \sigma}\left(q_{1}, q_{2}, q_{3}\right)=-i \int d^{4} x d^{4} y d^{4} z e^{-i\left(q_{1} \cdot x+q_{2} \cdot y+q_{3} \cdot z\right)}\left\langle 0\left|T\left\{j_{\mathrm{em}}^{\mu}(x) j_{\mathrm{em}}^{v}(y) j_{\mathrm{em}}^{\lambda}(z) j_{\mathrm{em}}^{\sigma}(0)\right\}\right| 0\right\rangle .
$$

Gauge invariance requires the HLbL tensor to satisfy the Ward-Takahashi (WT) identities

$$
\left\{q_{1}^{\mu}, q_{2}^{v}, q_{3}^{\lambda}, q_{4}^{\sigma}\right\} \Pi_{\mu v \lambda \sigma}\left(q_{1}, q_{2}, q_{3}\right)=0,
$$

where $q_{4}=q_{1}+q_{2}+q_{3}$. The HLbL tensor can be written a priori in terms of 138 basic Lorentz structures built out of the metric tensor and the four-momenta [34]. Our first task is to write the HLbL tensor in terms of Lorentz structures that satisfy the WT identities, while at the same time the scalar functions that multiply these structures must be free of kinematic singularities and zeros. A recipe for the construction of these structures has been given by Bardeen, Tung [35], and Tarrach [36] (BTT) for generic photon amplitudes. Gauge invariance imposes 95 linear relations between the 138 initial scalar functions. A generating set ${ }^{2}$ consisting of 43 elements can be constructed following Bardeen and Tung [35]. However, as it was shown by Tarrach [36], such a set is not free of kinematic singularities and has to be supplemented by additional structures. We find a redundant BTT generating set of dimension 54:

$$
\Pi^{\mu \nu \lambda \sigma}\left(q_{1}, q_{2}, q_{3}\right)=\sum_{i=1}^{54} T_{i}^{\mu \nu \lambda \sigma} \Pi_{i}(s, t, u),
$$

with scalar functions $\Pi_{i}$ depending on the Mandelstam variables $s=\left(q_{1}+q_{2}\right)^{2}, t=\left(q_{1}+q_{3}\right)^{2}$, $u=\left(q_{2}+q_{3}\right)^{2}$ as well as the photon virtualities $q_{i}^{2}$, and Lorentz structures $T_{i}^{\mu v \lambda \sigma}[25,26,39]$. This decomposition fulfills gauge invariance manifestly

$$
\left\{q_{1}^{\mu}, q_{2}^{v}, q_{3}^{\lambda}, q_{4}^{\sigma}\right\} T_{\mu \nu \lambda \sigma}^{i}=0,
$$

is highly crossing symmetric (with only 7 distinct structures, all remaining 47 being related to these by crossing transformations), and ensures that the coefficient functions $\Pi_{i}$ do not contain kinematic singularities and zeros. In addition, the BTT decomposition typically allows for a very economical representation of HLbL amplitudes, e.g. one of the structures coincides with the amplitude for a pseudoscalar pole, while even the SQED amplitude becomes very compact once expressed in terms of BTT functions.

\footnotetext{
${ }^{2}$ In 4 space-time dimensions, there are two additional linear relations, hence a basis consists of 41 elements [37, 38].
} 

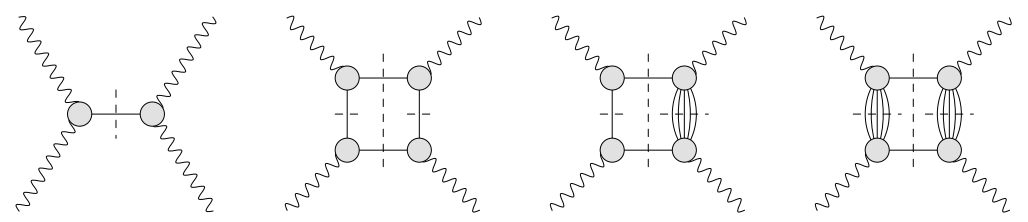

Figure 1: Unitarity diagrams according to the Mandelstam representation. Crossed diagrams are omitted.

\section{HLbL contribution to $(g-2)_{\mu}$}

The HLbL contribution to $a_{\mu}=(g-2)_{\mu} / 2$ can be extracted with the help of well-known Dirac projector techniques [40]. With our decomposition of the HLbL tensor in 54 structures, this amounts to the calculation of the following two-loop integral:

$$
\begin{aligned}
a_{\mu}^{\mathrm{HLbL}}= & -\frac{e^{6}}{48 m_{\mu}} \int \frac{d^{4} q_{1}}{(2 \pi)^{4}} \frac{d^{4} q_{2}}{(2 \pi)^{4}} \frac{1}{q_{1}^{2} q_{2}^{2}\left(q_{1}+q_{2}\right)^{2}} \frac{1}{\left(p+q_{1}\right)^{2}-m_{\mu}^{2}} \frac{1}{\left(p-q_{2}\right)^{2}-m_{\mu}^{2}} \\
& \times \operatorname{Tr}\left(\left(\not p+m_{\mu}\right)\left[\gamma_{\rho}, \gamma_{\sigma}\right]\left(\not p+m_{\mu}\right) \gamma_{\mu}\left(\not p+\not q_{1}+m_{\mu}\right) \gamma_{\lambda}\left(\not p-q_{2}+m_{\mu}\right) \gamma_{v}\right) \\
& \times\left.\sum_{i=1}^{54}\left(\frac{\partial}{\partial q_{4} \rho} T_{i}^{\mu \nu \lambda \sigma}\left(q_{1}, q_{2}, q_{4}-q_{1}-q_{2}\right)\right)\right|_{q_{4}=0} \prod_{i}\left(q_{1}, q_{2},-q_{1}-q_{2}\right) .
\end{aligned}
$$

After a Wick rotation of the momenta, five of the eight loop integrals can be carried out with the technique of Gegenbauer polynomials [41]. We have checked that this Wick rotation is justified even in the presence of anomalous thresholds in the scalar functions $\Pi_{i}$. In analogy to the pionpole contribution [42], a three-dimensional integral representation for the full HLbL contribution to $(g-2)_{\mu}$ can be worked out [27] :

$$
a_{\mu}^{\mathrm{HLbL}}=\frac{\alpha^{3}}{432 \pi^{2}} \int_{0}^{\infty} d \Sigma \Sigma^{3} \int_{0}^{1} d r r \sqrt{1-r^{2}} \int_{0}^{2 \pi} d \phi \sum_{i=1}^{12} T_{i}\left(Q_{1}, Q_{2}, Q_{3}\right) \bar{\Pi}_{i}\left(Q_{1}, Q_{2}, Q_{3}\right),
$$

where the $T_{i}$ are known kernel functions, the $\bar{\Pi}_{i}$ suitable linear combinations of the BTT $\Pi_{i}$, and the Euclidean momenta are given by [38]

$$
Q_{1,2}^{2}=\frac{\Sigma}{3}\left(1-\frac{r}{2} \cos \phi \mp \frac{r}{2} \sqrt{3} \sin \phi\right), \quad Q_{3}^{2}=\frac{\Sigma}{3}(1+r \cos \phi) .
$$

There are only 6 distinct functions $\bar{\Pi}_{i}$, the remaining ones are again related to these by crossing symmetry. It suffices to calculate the $\bar{\Pi}_{i}$ in the kinematic limit where $q_{4} \rightarrow 0$, the transition to $(g-2)_{\mu}$ then proceeds by means of Eq.(3.2).

\section{Mandelstam representation}

Although the scalar functions in the master formula Eq.(3.2) are needed only for the reduced kinematics where the limit $q_{4} \rightarrow 0$ is taken, we define the dispersion relation in the Mandelstam variables of the four-point function with general kinematics and evaluate it only afterwards for the special case $q_{4} \rightarrow 0$. This procedure has the following advantage: the HLbL contribution to $(g-2)_{\mu}$ splits into contributions from different topologies (shown in fig. 1), each of them linked 
to a specific sub-process, which is either data input or again a dispersively reconstructed quantity. These different contributions are discussed in the following.

Gauge invariance, encoded in the BTT decomposition, leads to Lorentz structures $T_{i}^{\mu \nu \lambda \sigma}$ of mass dimension 4,6 , and 8 . Hence, we expect the scalar functions $\Pi_{i}$ to be rather strongly suppressed at high energies. Thus we write down unsubtracted double-spectral (Mandelstam) representations for the $\Pi_{i}$ [43], i.e. parameter-free dispersion relations. The input to the dispersion relation are the residues at poles (due to single-particle intermediate states) and the discontinuities along branch cuts (due to two-particle intermediate states). Both are defined by the unitarity relation, in which the intermediate states are always on-shell. We neglect contributions from intermediate states consisting of more than two particles in the primary cut. Heavier intermediate states are expected to be suppressed by higher thresholds and smaller phase space, in agreement with the outcome of model calculations.

The first topology in fig. 1 consists of the pion pole, i.e. the terms arising from a single pion intermediate state. This contribution is well-known [42] and given by

$$
\begin{aligned}
& \bar{\Pi}_{1}^{\pi^{0} \text {-pole }}=-\frac{\mathscr{F}^{0} \gamma^{*} \gamma^{*}}{\left(-Q_{1}^{2},-Q_{2}^{2}\right) \mathscr{F}^{0} \pi^{*} \gamma^{*}} \\
& Q_{3}^{2}+M_{\pi}^{2} \\
& \bar{\Pi}_{2}^{\pi^{0} \text {-pole }}=-\frac{\mathscr{F}_{\pi^{0} \gamma^{*} \gamma^{*}}\left(-Q_{1}^{2},-Q_{3}^{2}\right) \mathscr{F}_{\pi^{0} \gamma^{*} \gamma^{*}}\left(-Q_{2}^{2}, 0\right)}{Q_{2}^{2}+M_{\pi}^{2}},
\end{aligned}
$$

where $\mathscr{F}^{0} \gamma^{*} \gamma^{*}$ denotes the pion transition form factor (for off-shell photons but an on-shell pion). For a dispersive determination of $a_{\mu}^{\pi^{0} \text {-pole }}$ we refer to [44].

The other topologies in fig. 1 are obtained by selecting two-pion intermediate states in the primary cut. The sub-process $\gamma^{*} \gamma^{*} \rightarrow \pi \pi$ is again cut in the crossed channel. If we single out the pion-pole contribution in both of the sub-processes, we obtain the box topologies for HLbL. For higher intermediate states in the crossed channel of $\gamma^{*} \gamma^{*} \rightarrow \pi \pi$, we obtain boxes with multi-particle cuts instead of poles in the sub-processes.

By explicitly constructing the Mandelstam representation, we have shown that the box topologies in the sense of unitarity have the same analytic structure as the one-loop sQED contribution, multiplied with pion electromagnetic form factors $F_{\pi}^{V}\left(q_{i}^{2}\right)$ for each of the off-shell photons (FsQED). The dispersion relation defines unambiguously this particular $q_{i}^{2}$ dependence. With the construction of the Mandelstam representation, we prove that FsQED and box topologies are the same. Note that the sQED loop contribution in terms of Feynman diagrams consists of boxes, triangles, and bulbs, but that the corresponding unitarity diagrams are just box topologies. This can be understood as follows: in SQED, the appearance of triangle and bulb diagrams is due to the seagull vertex, needed to ensure gauge invariance. In our formalism, gauge invariance is already encoded in the BTT tensor decomposition Eq.(2.3). Due to the high degree of crossing symmetry, the pion-box contribution can be expressed in terms of either fixed- $s,-t$, or $-u$ dispersion relations, or in a symmetrized form

$$
\Pi_{i}^{\pi \text {-box }}(s, t, u)=\frac{1}{3}\left[\frac{1}{\pi} \int_{4 M_{\pi}^{2}}^{\infty} d t^{\prime} \frac{\operatorname{Im} \Pi_{i}^{\pi-\operatorname{box}}\left(s, t^{\prime}, u^{\prime}\right)}{t^{\prime}-t}+\frac{1}{\pi} \int_{4 M_{\pi}^{2}}^{\infty} d u^{\prime} \frac{\operatorname{Im} \Pi_{i}^{\pi-\operatorname{box}}\left(s, t^{\prime}, u^{\prime}\right)}{u^{\prime}-u}+\text { fixed- } t+\text { fixed- } u\right] .
$$

In this case the representation is exact. 
Once heavier intermediate states are considered, see Fig. 1, a more detailed investigation of the double spectral functions is required. In practice, such contributions can be included using a partial-wave expansion, in which case the sub-process becomes a polynomial in the crossed variable and the crossed-channel cuts are neglected. Writing down all crossed versions of the unitarity diagrams in Fig. 1 with a two-particle primary cut, one sees that each double spectral region appears exactly twice in a symmetrized form as in (4.2), so that the prefactor changes according to $1 / 3 \rightarrow 1 / 2$ [39], with corrections suppressed by the mass scale of the neglected LHC. In particular, this representation becomes exact for $\pi \pi$-rescattering effects, which, by definition, are polynomial in the crossed Mandelstam variable.

For a numerical evaluation of the pion box contribution, the only input needed is the pion vector form factor in the space-like region. By fitting a dispersive representation which accounts for the prominent singularities in the low-energy region as suggested in $[45,46]$ to both spacelike [47] and time-like form factor data [48, 49, 50, 51, 52, 53], we obtain

$$
a_{\mu}^{\pi \mathrm{box}}=-15.9(2) \times 10^{-11}
$$

where the uncertainty is determined from the differences between the time-like data sets as well as the details of the fit representation. We stress that previous evaluations of a "pion loop" [54, 55] had large model-dependent uncertainties, whereas our evaluation of an unambiguously-defined pion box has a negligible one.

\section{Partial-wave expansion}

Constraints from unitarity are most conveniently formulated in a partial-wave expansion for HLbL helicity amplitudes $h_{\lambda_{1} \lambda_{2}, \lambda_{3} \lambda_{4}}^{J}$ with angular momentum $J$ and helicity labels $\lambda_{i}$. In this case the unitarity relation becomes diagonal

$$
\operatorname{Im} h_{\lambda_{1} \lambda_{2}, \lambda_{3} \lambda_{4}}^{J}(s)=\frac{\sigma_{\pi}(s)}{16 \pi S} h_{J, \lambda_{1} \lambda_{2}}(s) h_{J, \lambda_{3} \lambda_{4}}^{*}(s)
$$

where $\sigma_{\pi}(s)=\sqrt{1-4 M_{\pi}^{2} / s}, S=2$ for indistinguishable particles, and $h_{J, \lambda_{1} \lambda_{2}}$ the helicity partial waves for $\gamma^{*} \gamma^{*} \rightarrow \pi \pi$, and once formulated in isospin basis, Watson's theorem guarantees that the phases on the right-hand side cancel to produce a well-defined imaginary part.

There are 41 independent helicity amplitudes for the full HLbL tensor, which reduce to 27 if one photon is taken on-shell. By overcoming several technical and conceptual challenges [27, 39], we were able to construct a set of 27 amplitudes $\check{\Pi}_{i}$ related to the 27 singly-on-shell helicity amplitudes by a basis change that we have derived in explicit analytic form. In the limit $q_{4} \rightarrow 0$ a subset of the $\check{\Pi}_{i}$ includes all the scalar functions needed as input in (3.2) [39]. Moreover, this set of 27 amplitudes is manifestly free of Tarrach [36] or $d=4$ ambiguities [38]. For singly-onshell kinematics, there still exist 15 sum rules among the 27 helicity amplitudes, which we have exploited to optimize to a certain degree the representation with respect to the convergence of the partial-wave expansion.

The pion box provides an ideal test case for our partial-wave framework since the full result is known and explicit expressions for all BTT scalar functions are available. Our results, for simplicity obtained by using a vector-meson-dominance pion form factor $F_{\pi}^{V}\left(q^{2}\right)=M_{\rho}^{2} /\left(M_{\rho}^{2}-q^{2}\right)$, 
Table 1: Saturation of $a_{\mu}^{\pi \text {-box }}$ for maximal angular momentum $J_{\max }$.

\begin{tabular}{lllll}
\hline$J_{\max }$ & fixed- $s$ & fixed- $t$ & fixed- $u$ & average \\
\hline 0 & $0.0 \%$ & $106.2 \%$ & $106.2 \%$ & $70.8 \%$ \\
2 & $73.9 \%$ & $102.3 \%$ & $92.7 \%$ & $89.6 \%$ \\
4 & $89.2 \%$ & $101.5 \%$ & $96.4 \%$ & $95.7 \%$ \\
6 & $94.3 \%$ & $100.7 \%$ & $97.9 \%$ & $97.6 \%$ \\
8 & $96.5 \%$ & $100.4 \%$ & $98.7 \%$ & $98.5 \%$ \\
\hline
\end{tabular}

with $a_{\mu}^{\pi \text {-box, VMD }}=-16.4 \times 10^{-11}$, are shown in Table 1 , demonstrating that each fixed- $s,-t,-u$ representation approaches the full result (going up to $J_{\max }=20$, we checked that also the remaining differences disappear after partial-wave resummation). The vanishing $S$-wave contribution for fixed-s is well understood and partly a matter of convention [27]. The convergence pattern looks very reasonable.

\section{6. $\pi \pi$ rescattering effects}

The partial-wave decomposition of the pion box demonstrates that the traditional SQED pion loop can be understood as the result of resumming the Born-term contributions to the $\gamma^{*} \gamma^{*} \rightarrow \pi \pi$ partial waves $h_{J, \lambda_{1} \lambda_{2}}(s)$ introduced in the context of the unitarity relation (5.1). However, unitarity for the sub-process itself implies

$$
\operatorname{Im} h_{J, \lambda_{1} \lambda_{2}}^{I}(s)=\sin \delta_{J}^{I}(s) e^{-i \delta_{J}^{I}(s)} h_{J, \lambda_{1}}^{I} \lambda_{2}(s),
$$

with isospin labels $I$ and $\pi \pi$ phase shifts $\delta_{J}^{I}$. This relation is clearly violated for the (real) Born terms alone. With a partial-wave framework at our disposal, we are thus in the position to evaluate the corresponding unitarity corrections, more conventionally referred to as $\pi \pi$-rescattering effects, as a first important step to account for contributions beyond the pion box.

In contrast to the on-shell and singly-virtual case [28, 29, 30], the calculation of the $\gamma^{*} \gamma^{*} \rightarrow \pi \pi$ partial waves for two off-shell photons is complicated by the fact that even for $S$-waves two different helicity partial waves, $h_{0,++}$ and $h_{0,00}$, become coupled, including off-diagonal kernel functions required to eliminate kinematic singularities $[22,26]$. We applied this framework to construct the $\gamma^{*} \gamma^{*} \rightarrow \pi \pi$ amplitudes that correspond to the rescattering corrections to the Born terms, whose solution can still be derived based on Muskhelishvili-Omnès methods [56, 57]. We used $\pi \pi$ phase shifts based on the modified inverse-amplitude method [58], for the main reason that it has a simple analytic expression, while at the same time it reproduces accurately the low-energy properties of the phase shifts as well as pole position and couplings of the $f_{0}(500)$ resonance [27]. We also tested the sensitivity to the asymptotic part of the dispersive integrals by studying solutions with different cutoff values $\Lambda=[1 \mathrm{GeV}, \infty)$, constructed with finite-matching-point techniques [59, 29, 60, 61, 62]. Our results for the rescattering contribution, summarized in Table 2, turn out to be stable over a wide range of cutoffs, indicating that our input for the $\gamma^{*} \gamma^{*} \rightarrow \pi \pi$ partial waves reliably unitarizes the Born-term LHC, which should indeed dominate at low energies. The isospin- 0 part of the result 
Table 2: $S$-wave rescattering corrections to $a_{\mu}^{\pi \text {-box }}$, in units of $10^{-11}$, for both isospin components and in total.

\begin{tabular}{lrrrr} 
cutoff & $1 \mathrm{GeV}$ & $1.5 \mathrm{GeV}$ & \multicolumn{1}{l}{$2 \mathrm{GeV}$} & \multicolumn{1}{l}{$\infty$} \\
\hline$I=0$ & -9.2 & -9.5 & -9.3 & -8.8 \\
$I=2$ & 2.0 & 1.3 & 1.1 & 0.9 \\
sum & -7.3 & -8.3 & -8.3 & -7.9 \\
\hline
\end{tabular}

can be interpreted as a model-independent implementation of the contribution from the $f_{0}(500)$ of about $-9 \times 10^{-11}$ to HLbL scattering in $(g-2)_{\mu}$. In total, we obtain for the $\pi \pi$-rescattering effects related to the pion-pole $\mathrm{LHC}$

$$
a_{\mu, J=0}^{\pi \pi, \pi \text {-pole LHC }}=-8(1) \times 10^{-11},
$$

where the error is dominated by the uncertainties related to the asymptotic parts of the integral.

Finally, it is instructive to consider the separate contributions not in the isospin, but in the charge basis. Our numerical analysis [27] shows that the rescattering effects in (6.2) are, as expected, dominated by the charged pion, with input for the $\gamma^{*} \gamma^{*} \rightarrow \pi \pi$ partial waves fully consistent both with the recent COMPASS measurement [63] of its dipole polarizability and the corresponding two-loop chiral prediction [64]. For this reason (6.2) can be considered a model-independent implementation of effects related to the low-energy constants $L_{9}$ and $L_{10}$ in Chiral Perturbation Theory, which were suspected to produce large effects in [32]. Our calculation proves that this is not the case, and that the related rescattering corrections are indeed of much more reasonable size (a similar conclusion was reached within a model approach in [33]).

To summarize, we have shown that our framework allows us to estimate very accurately the combined effect of two-pion intermediate states generated by a pion-pole LHC and its $S$-wave unitarization

$$
a_{\mu}^{\pi \text {-box }}+a_{\mu, J=0}^{\pi \pi, \pi \text {-pole LHC }}=-24(1) \times 10^{-11},
$$

which is considered to be among the most important contributions after the dominant pseudoscalar poles, but was so far affected by significant uncertainties. This first numerical result based on our dispersive approach lays the foundation for extensions towards higher partial waves, an improved LHC in the $\gamma^{*} \gamma^{*} \rightarrow \pi \pi$ subamplitudes as well as higher-mass intermediate states, all important prerequisites for a model-independent data-driven evaluation of the complete HLbL contribution to $(g-2)_{\mu}$.

The speaker (MP) thanks the conveners of the section QCD and New Physics of the XIII Quark Confinement and the Hadron Spectrum conference for their kind invitation. Financial support by the DFG (SFB/TR 16, "Subnuclear Structure of Matter," SFB/TR 110, "Symmetries and the Emergence of Structure in QCD"), the DOE (Grant No. DE-FG02-00ER41132 and DE-SC0009919), the National Science Foundation (Grant No. NSF PHY-1125915), and the Swiss National Science Foundation (Project No. P300P2-167751) is gratefully acknowledged. 


\section{References}

[1] G. W. Bennett et al. [Muon g-2 Collaboration], Phys. Rev. D 73 (2006) 072003 [hep-ex/0602035].

[2] T. Blum, A. Denig, I. Logashenko, E. de Rafael, B. Lee Roberts, T. Teubner and G. Venanzoni, arXiv:1311.2198 [hep-ph].

[3] J. Grange et al. [Muon g-2 Collaboration], arXiv:1501.06858 [physics.ins-det].

[4] N. Saito [J-PARC g-'2/EDM Collaboration], AIP Conf. Proc. 1467 (2012) 45.

[5] F. Jegerlehner and A. Nyffeler, Phys. Rept. 477 (2019) 1 [arXiv:0902.3360 [hep-ph]].

[6] J. Prades, E. de Rafael and A. Vainshtein, Adv. Ser. Direct. High Energy Physics 20 (2009) 303 [arXiv:0901.0306 [hep-ph]].

[7] F. Jegerlehner, Springer Tracts Mod. Physics 274 (2017) 1.

[8] F. Jegerlehner, EPJ Web Conf. 166 (2018) 00022 [arXiv:1705.00263 [hep-ph]].

[9] M. Davier, A. Hoecker, B. Malaescu and Z. Zhang, Eur. Phys. J. C77 (2017) 827 [arXiv:1706.09436 [hep-ph]].

[10] A. Keshavarzi, D. Nomura and T. Teubner, Phys. Rev. D97 (2018) 114025 [arXiv:1802.02995 [hep-ph]].

[11] G. Colangelo, M. Hoferichter and P. Stoffer, [arXiv:1810.00007 [hep-ph]].

[12] M. K. Marinkovic, Few Body Syst. 58 (2017) 140 [arXiv:1704.06425 [hep-ph]].

[13] M. Hayakawa, T. Blum, T. Izubuchi and N. Yamada, PoS LAT 2005 (2006) 353 [hep-lat/0509016].

[14] T. Blum, M. Hayakawa and T. Izubuchi, PoS LATTICE 2012 (2012) 022 [arXiv:1301.2607 [hep-lat]].

[15] T. Blum, S. Chowdhury, M. Hayakawa and T. Izubuchi, Phys. Rev. Lett. 114 (2015) 1, 012001 [arXiv:1407.2923 [hep-lat]].

[16] J. Green, O. Gryniuk, G. von Hippel, H. B. Meyer and V. Pascalutsa, arXiv:1507.01577 [hep-lat].

[17] T. Blum, N. Christ, M. Hayakawa, T. Izubuchi, L. Jin and C. Lehner, Phys. Rev. D 93, 014503 (2016) [arXiv:1510.07100 [hep-lat]].

[18] T. Blum, N. Christ, M. Hayakawa, T. Izubuchi, L. Jin, C. Jung and C. Lehner, Phys. Rev. Lett. 118 , 022005 (2017) [arXiv:1610.04603 [hep-lat]].

[19] T. Blum, N. Christ, M. Hayakawa, T. Izubuchi, L. Jin, C. Jung and C. Lehner, Phys. Rev. D 96 (2017) no.3, 034515 [arXiv:1705.01067 [hep-lat]].

[20] A. Gérardin, H. B. Meyer and A. Nyffeler, Phys. Rev. D 94, 074507 (2016) [arXiv:1607.08174 [hep-lat]].

[21] N. Asmussen et al., EPJ Web Conf. 179 (2018) 01017 [arXiv:1801.04238 [hep-lat]].

[22] G. Colangelo, M. Hoferichter, M. Procura and P. Stoffer, JHEP 1409 (2014) 091 [arXiv:1402.7081 [hep-ph]].

[23] G. Colangelo, M. Hoferichter, B. Kubis, M. Procura and P. Stoffer, Phys. Lett. B 738 (2014) 6 [arXiv:1408.2517 [hep-ph]].

[24] V. Pauk and M. Vanderhaeghen, Phys. Rev. D 90 (2014) 11, 113012 [arXiv:1409.0819 [hep-ph]].

[25] P. Stoffer, arXiv:1412.5171 [hep-ph]. 
[26] G. Colangelo, M. Hoferichter, M. Procura and P. Stoffer, JHEP 1509 (2015) 074 [arXiv:1506.01386 [hep-ph]].

[27] G. Colangelo, M. Hoferichter, M. Procura and P. Stoffer, Phys. Rev. Lett. 118, 232001 (2017) [arXiv:1701.06554 [hep-ph]].

[28] R. García-Martín and B. Moussallam, Eur. Phys. J. C 70, 155 (2010) [arXiv:1006.5373 [hep-ph]].

[29] M. Hoferichter, D. R. Phillips and C. Schat, Eur. Phys. J. C 71, 1743 (2011) [arXiv:1106.4147 [hep-ph]].

[30] B. Moussallam, Eur. Phys. J. C 73, 2539 (2013) [arXiv:1305.3143 [hep-ph]].

[31] K. T. Engel, H. H. Patel and M. J. Ramsey-Musolf, Phys. Rev. D 86, 037502 (2012) [arXiv:1201.0809 [hep-ph]].

[32] K. T. Engel and M. J. Ramsey-Musolf, Phys. Lett. B 738, 123 (2014) [arXiv:1309.2225 [hep-ph]].

[33] J. Bijnens and J. Relefors, JHEP 1609, 113 (2016) [arXiv:1608.01454 [hep-ph]].

[34] R. A. Leo, A. Minguzzi and G. Soliani, Nuovo Cim. A 30 (1975) 270.

[35] W. A. Bardeen and W. K. Tung, Phys. Rev. 173 (1968) 1423 [Phys. Rev. D 4 (1971) 3229].

[36] R. Tarrach, Nuovo Cim. A 28 (1975) 409.

[37] G. Eichmann, C. S. Fischer, W. Heupel and R. Williams, arXiv:1411.7876 [hep-ph].

[38] G. Eichmann, C. S. Fischer and W. Heupel, Phys. Rev. D 92 (2015) 5, 056006 [arXiv:1505.06336 [hep-ph]].

[39] G. Colangelo, M. Hoferichter, M. Procura, P. Stoffer, JHEP 1704 (2017) 161 [arXiv:1702.07347 [hep-ph]].

[40] J. Aldins, T. Kinoshita, S. J. Brodsky and A. J. Dufner, Phys. Rev. D 1 (1970) 2378.

[41] J. L. Rosner, Annals Phys. 44 (1967) 11.

[42] M. Knecht and A. Nyffeler, Phys. Rev. D 65 (2002) 073034 [hep-ph/0111058].

[43] S. Mandelstam, Phys. Rev. 112 (1958) 1344.

[44] M. Hoferichter, B.-L. Hoid, B. Kubis, S. Leupold and S. P. Schneider JHEO 1810 (2018) 141.

[45] H. Leutwyler, hep-ph/0212324.

[46] G. Colangelo, Nucl. Phys. Proc. Suppl. 131 (2004) 185 [hep-ph/0312017].

[47] S. R. Amendolia et al. [NA7 Collaboration], Nucl. Phys. B 277, 168 (1986).

[48] M. N. Achasov et al., J. Exp. Theor. Phys. 103, 380 (2006) [Zh. Eksp. Teor. Fiz. 130, 437 (2006)] [hep-ex/0605013].

[49] R. R. Akhmetshin et al. [CMD-2 Collaboration], Phys. Lett. B 648, 28 (2007) [hep-ex/0610021].

[50] B. Aubert et al. [BaBar Collaboration], Phys. Rev. Lett. 103, 231801 (2009) [arXiv:0908.3589 [hep-ex]].

[51] F. Ambrosino et al. [KLOE Collaboration], Phys. Lett. B 700, 102 (2011) [arXiv:1006.5313 [hep-ex]].

[52] D. Babusci et al. [KLOE Collaboration], Phys. Lett. B 720, 336 (2013) [arXiv:1212.4524 [hep-ex]].

[53] M. Ablikim et al. [BESIII Collaboration], Phys. Lett. B 753, 629 (2016) [arXiv:1507.08188 [hep-ex]]. 
[54] J. Bijnens, E. Pallante and J. Prades, Phys. Rev. Lett. 75, 1447 (1995) Erratum: [Phys. Rev. Lett. 75, 3781 (1995)] [hep-ph/9505251].

[55] M. Hayakawa, T. Kinoshita and A. I. Sanda, Phys. Rev. Lett. 75, 790 (1995) [hep-ph/9503463].

[56] N. I. Muskhelishvili, Singular Integral Equations, Wolters-Noordhoff Publishing, Groningen, 1953 [Dover Publications, 2nd edition, 2008].

[57] R. Omnès, Nuovo Cim. 8, 316 (1958).

[58] A. Gómez Nicola, J. R. Peláez and G. Ríos, Phys. Rev. D 77, 056006 (2008) [arXiv:0712.2763 [hep-ph]].

[59] P. Büttiker, S. Descotes-Genon and B. Moussallam, Eur. Phys. J. C 33, 409 (2004) [hep-ph/0310283].

[60] C. Ditsche, M. Hoferichter, B. Kubis and U.-G. Meißner, JHEP 1206, 043 (2012) [arXiv:1203.4758 [hep-ph]].

[61] M. Hoferichter, C. Ditsche, B. Kubis and U.-G. Meißner, JHEP 1206, 063 (2012) [arXiv:1204.6251 [hep-ph]].

[62] M. Hoferichter, J. Ruiz de Elvira, B. Kubis and U.-G. Meißner, Phys. Rept. 625, 1 (2016) [arXiv:1510.06039 [hep-ph]].

[63] C. Adolph et al. [COMPASS Collaboration], Phys. Rev. Lett. 114, 062002 (2015) [arXiv:1405.6377 [hep-ex]].

[64] J. Gasser, M. A. Ivanov and M. E. Sainio, Nucl. Phys. B 745, 84 (2006) [hep-ph/0602234]. 